\title{
Stroke and Alcohol Poisoning Mortality in Russia
}

\author{
Y. E. Razvodovsky \\ Grodno State Medical University, Belarus, Grodno, str. Gorky 80, 230008, \\ *Corresponding Author: razvodovsky@tut.by
}

Copyright (C) 2013 Horizon Research Publishing All rights reserved.

\begin{abstract}
Abtract Background: Stroke is an international health problem with high associated human and economic costs. Stroke burden is expected to be rising, especially in developing countries. The mortality rate from stroke in Russia is one of the highest in the world. Epidemiological evidence suggests that binge drinking is an important determinant of high stroke mortality rate in Russia. In line with this evidence we assume that devastating combination of higher level of alcohol consumption per capita and binge drinking of vodka results in a close association between binge drinking and stroke mortality at the aggregate level in Russia. This paper was design to estimate the aggregate level effect of binge drinking on stroke mortality rate in Russian. Method: Trends in age-adjusted, sex-specific stroke mortality and fatal alcohol poisoning rate (as a proxy for binge drinking) from 1965 to 2005 were analyzed employing a distributed lags analysis in order to asses bivariate relationship between the two time series. Results: The results of the time series analysis indicates the presence of a statistically significant association between the two time series at zero lag for male $(r=0.76$; S.E. $=0.17)$, and for female $(r=0.64$; S.E. $=0.17)$. Conclusion: The results of present study support the hypothesis that alcohol played a crucial role in cerebrovascular mortality fluctuation in Russia over the past decades. This study also indicates that substantial proportion of stroke deaths in Russia is due to acute effect of binge drinking. The findings from the present study have important implications as regards cerebrovascular mortality prevention indicating that a restrictive alcohol policy can be considered as an effective measure of prevention in countries where higher rate of alcohol consumption and binge drinking pattern.
\end{abstract}

Keywords Stroke, Alcohol Poisoning, Time-Series Analysis, Russia, 1965-2005.

\section{Introduction}

Stroke is one of the major cause of death in the developed world and a top ten contributor to the global burden of disease (Donna, Fisher, Macleod \& Davis, 2007). Stroke burden is expected to be rising, especially in developing countries (Kim \& Johnston, 2011). In relation to this, identification of the risk factors is a high priority front the public health perspective.

Alcohol has been identified as both a risk and a protective factor for stroke (Gorelic, 1987; Sacco, Elkind, Boden-Albala, Lin, Kargman, Hauser, Shea \& Paik, 1999). Accumulated research evidence suggests that heavy alcohol consumption increases the relative risk of stroke, while light or moderate alcohol consumption may be protective against ischemic stroke (Djousse, Ellison, Beiser, Scaramucci, D'Agostino \& Wolf, 2002; Patra, Taylor, Irving, Roerecke, Baliunas, Mohapatra \& Rehm, 2010). The meta-analysis of 35 observational studies published between 1966 and 2002 revealed that compared with abstainers, consumption of more than $60 \mathrm{~g}$ of alcohol per day was associated with an increased relative risk of total stroke, 1.64 (95\% CI, 1.39-1.93), ischemic stroke, 1.69 (95\% CI, 1.34-2.15), and hemorrhagic stroke, $2.18 \quad(95 \%$ CI, 1.48-3.20) (Reynolds, Lewis, Nolen, Kinney, Sathya \& He, 2003).

There is evidence that pattern of drinking may have modifying influence on stroke risk independently of the amount of alcohol consumed. Previous findings indicated an acute detrimental effect of binge drinking as a trigger of stroke. For instance, a Finish case-control study reported that consumption of 151 to $300 \mathrm{~g}$ and $>300 \mathrm{~g}$ of alcohol within the week before stroke onset is associated with a significantly higher risk of stroke with adjusted OR of 3.6 (95\% CI 1.7 to 7.8$)$ and $3.7(95 \% \mathrm{CI}, 1.6$ to 8.7$)$ respectively (Hillbom, Numminen \& Juvela, 1999). A Swedish cohort study reported a higher risk of ischemic stroke among occasional heavy drinkers than among life-time abstainers (Romelsjo \& Leifman, 1999). In the prospective cohort study binge drinking pattern was an independent risk factor for all strokes (Sundell, Salomaa, Vartiainen, Poikolainen \& Laatikainen, 2008). Comparing with non-binge drinkers, the hazard ratio for total stroke among binge drinkers was 1.85 (95\% CI, 1.35 to 2.54$)$ after adjusting for average alcohol consumption and other potential confounders. Furthermore, heavy drinking within 24 hours before the onset of stroke was found to be a risk 
factor for ischemic stroke due to cardiogenic embolism (Mostofsky, Burger, Schlaug, Mukamal, Rosamond \& Mittleman, 2010).

Russia has one of the world's highest stroke incidence and mortality rates (Kim \& Johnston, 2011). In contrast to Western Europe (WE), where stroke rates are falling, in Russia stroke is rising cause of disability and deaths (Kesteloot, Sans \& Kromhout, 2006; Muller-Nordhorn, Binting, Roll \& Willich, 2008; Rieks, Willich, Mueller-Riemenschneider, 2012). The higher rates of stroke mortality observed in Russia as compared with WE were in the young and middle-age groups. In 2002 the stroke mortality rate in Russia among men age $45-54$ years was ten times higher than in France, Germany or Italy (Kim \& Johnston, 2011). It was concluded that these differences can only be partly accounted for by differences in quality of stroke care throughout Europe and might be attributed to different prevalence for risk factors (Ginter, 1995; Pajak \& Kozela, 2012; Petruchin \& Lunina, 2012). In a population-based case-control study hypertension, ischemic heart disease, smoking and high body mass index were major risk factors for ischemic stroke in the Russian population (Feigin, Wiebers, Nikitin, O'Fallon \& Whisnant, 1998).

Several studies have emphasized the importance of binge drinking as important determinant of high stroke mortality rate in Russia. In particular, the results of a case-control study suggest that recent episodes of heavy drinking is associated with substantial increases in stroke mortality independently of long-term hazardous drinking (Shkolnikov, McKee, Chervyakov \& Kyrianov, 2002). More recent case-control study from western Siberia reported a positive association of stroke risk with amount of alcohol drunk on single occasion (Leon, Saburova, Tomkins, Andreev, Kiryanov, McKee \& Shkolnikov, 2007).

The level of alcohol consumption in Russia is among the highest in the world with an annual sales rate about 10 litres of pure alcohol per capita, while independent estimates show a figure as high as 17 litres (Nemtsov \& Razvodovsky, 2008).The distinctive trait of Russian drinking culture is the preference for binge drinking of vodka, leading to an increase in deaths from alcohol poisoning and cardiovascular diseases (Nemtsov, 2002; Nemtsov \& Razvodovsky, 2008; Stickley, Leinsalu, Andreew, Razvodovsky, Vagero. \& McKee, 2007). In line with this evidence we assume that devastating combination of higher level of alcohol consumption per capita and binge drinking of vodka results in a close association between binge drinking and stroke mortality at the aggregate level in Russia.

The aim of the present study was to estimate effect of binge drinking on stroke mortality rate in Russian using aggregate-level data of stroke mortality and fatal alcohol poisoning rates.

\section{Methods}

\subsection{Data}

The data on age-adjusted sex-specific stroke and alcohol poisoning mortality rates per 1000.000 of the population are taken from the Russian State Statistical Committee (Rosstat). It should be noted that in Russia the fatal alcohol poisoning rate is likely to be a good measure of heavy episodic drinking (Stickley, et al., 2007). Therefore, a common approach is to use the fatal alcohol poisoning rate as an indicator of binge drinking in these countries (Razvodovsky, 2009).

\subsection{Statistical analysis}

The statistical analysis was performed using the package "Statistica". It is generally agreed that bivariate correlations between the two raw time-series are spurious due to common sources of trends and autocorrelation (Norstrom \& Skog, 2001). Therefore in order to reduce the risk of obtaining a spurious relation between two variables that have common trends, the trends should be removed by means of a differencing procedure: $\nabla x_{t}=x_{t}-x_{t-1}$ This means analyzing annual changes rather than raw data. The process of removing systematic variation within time series prior to the examination of potential causal relationships is referred to as "prewhitening" (Box a \& Jenkins, 1976). A further step entails the inspection of the cross-correlation function in order to estimate the association between the two prewhitened time series. We used an unconstrained polynomial distributed lags analysis to estimate the relationship between the time series fatal alcohol poisoning (independent variable) and stroke mortality (dependent variable) rates in this paper.

\section{Results}

According to official statistics, the male stroke mortality rate increased 1.5 times (from 2990.4 to 4492.5 per 1000.000 of residents) and female mortality rate increased 1.3 times (from 2640.6 to 3464.3 per 1000.000 of population) in Russia from 1965 to 2005 . The fatal alcohol poisoning rate for male increased 2.7 times (from 172.7 to 468.7 per 1000.000 of residents), and for female increased 4.6 times (from 24.2 to 113.6 per 1000.000 of population). The graphical evidence suggests that the trends in both stroke and alcohol poisoning mortality for male and female seem to follow each other across the 1965-2005 time series. The two time series fluctuated over the period for both sexes: increased steadily from 1965 to 1984, then dropped sharply in 1985-1988, began to increase in 1988-1989, dramatically jumped from 1991 to 1994 . From 1995 there was a fall in the rates until 1998 when it again began to rise and than started to decrease in the last years

The Spearman's correlation analysis suggests a strong positive association between the two variables for male $(\mathrm{r}=0.71 ; \mathrm{p}=0.000)$ and female $(\mathrm{r}=0.67 ; \mathrm{p}=0.000)$. As can be 
seen from Figures 1-2 there are linear and S-shape trends in the time series. These trends were removed by means of first-order differencing procedure. After pre-whitening the cross-correlations between fatal alcohol poisoning and stroke mortality time series were inspected. The outcome indicated statistically significant cross-correlation between the two variables for male $(\mathrm{r}=0.76$; $\mathrm{SE}=0.17)$ and female $(r=0.64 ; \mathrm{SE}=0.17)$ at zero lag. The results of the distributed lags analysis also suggest that only contemporaneous correlation (lag 0 ) is statistically significant for both males and females (tables 1-2).

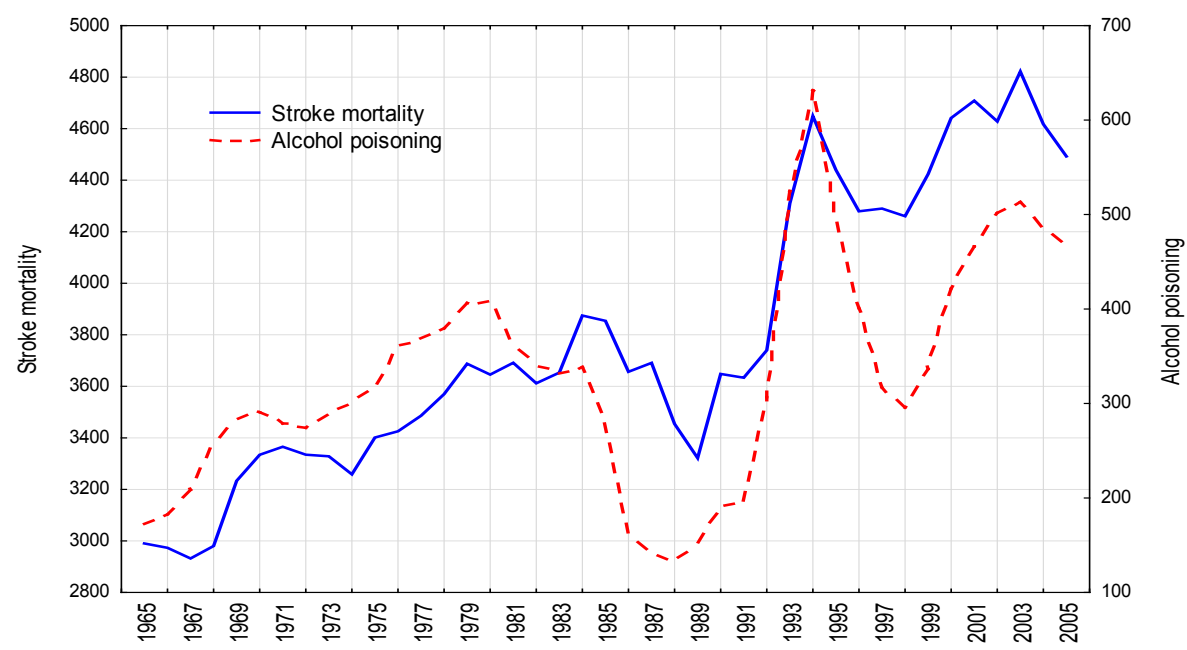

Figure 1. Trends in male fatal alcohol poisoning and stroke mortality rates in Russia between 1965 and 2005 .

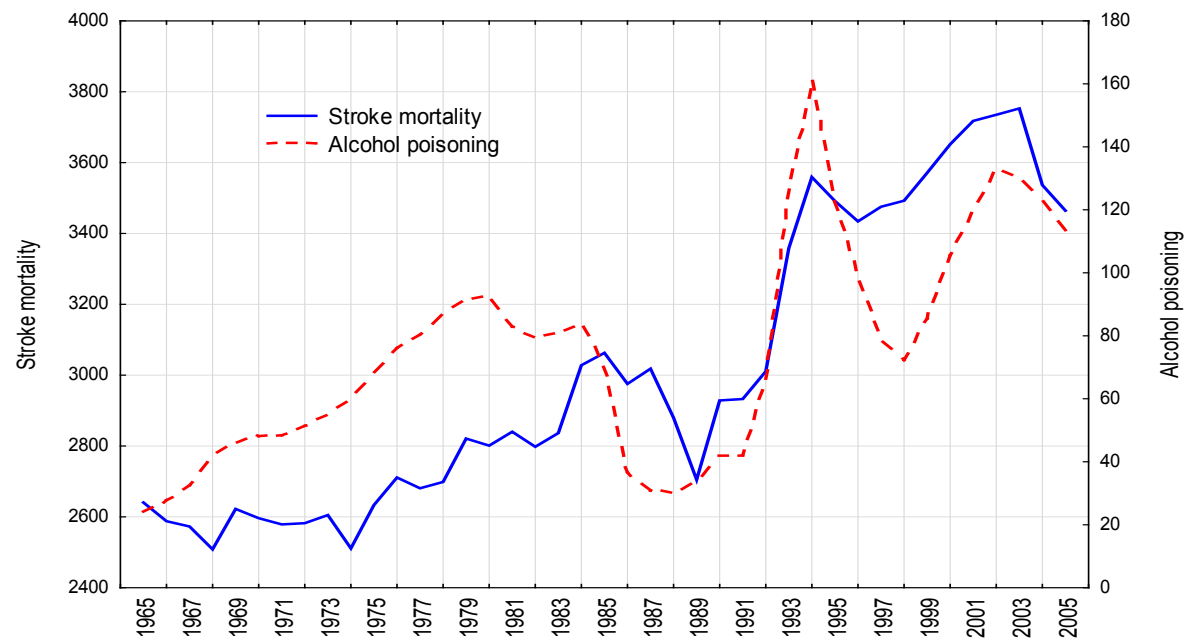

Figure 2. Trends in female fatal alcohol poisoning and stroke mortality rates in Russia between 1965 and 2005 .

Table 1. The results of distributed lags analysis for males.

\begin{tabular}{|c|c|c|c|c|}
\hline Lags & Regressn Coeff & Standard Error & $\mathrm{t}$ & $\mathrm{p}$ \\
\hline 0 & 3,88 & 1,15 & 3,35 & 0,000 \\
\hline 1 & 0,46 & 1,32 & 0,34 & 0,731 \\
\hline 2 & 0,07 & 1,16 & 0,06 & 0,946 \\
\hline
\end{tabular}

Table 2. The results of distributed lags analysis for females.

\begin{tabular}{|c|c|c|c|c|}
\hline Lags & Regressn Coeff & Standard Error & $\mathrm{t}$ & $\mathrm{p}$ \\
\hline 0 & 1,83 & 0,43 & 4,27 & 0,000 \\
\hline 1 & 0,31 & 0,50 & 0,61 & 0,543 \\
\hline 2 & $-0,13$ & 0,43 & $-0,31$ & 0,753 \\
\hline
\end{tabular}




\section{Discussion}

The time series analysis suggests a positive relationship between fatal alcohol poisoning and stroke mortality at zero lag. We argue that in this case independent variable is directly influencing the dependent variable and that there is no evidence of a lagged relationship between the two time series. It is important to point out, that the size of the bivariate association between fatal alcohol poisoning as a proxy for binge drinking and stroke deaths for men is substantially greater than for women. The harmful drinking pattern might be responsible for the gender differences in alcohol poisoning and stroke mortality association. The population surveys from Russia show consistently higher rates of binge drinking among men than women. Indeed, the results of study carried out in Archangelsk suggest that $61.9 \%$ of male and $25.7 \%$ of female industrial workers had a consumption pattern that was hazardous according to the AUDIT definition (Nilssen, Averina, Brenn, Brox, Kalinin, Archipovski, 2005).

The experiences of Russia during the last decades present a remarkable opportunity to examine the relationship between social processes and cerebrovascular mortality. As we can see, stroke deaths and fatal alcohol poisoning trends have been more or less correlated with the great societal transformation. The sharp decline in the time series in the mid-1980s corresponds with the anti-alcohol campaigns by Gorbachev, which significantly reduced alcohol consumption by limiting its manufacture and availability. The dramatic increases in the two time trends in the early 1990s corresponds to dissolution of the Soviet Union and the profound socio-economic and political changes occurring during the transitional period to the post communism. It seems plausible, that alcohol poisoning and stroke mortality rates increase observed in the yearly 1990s was the direct consequence of abolishing the state alcohol monopoly in 1992. Moreover, there is indirect evidence that mortality fluctuations during the 1990s may be related to changes in alcohol availability. When price of alcohol fell relative to basic foods stuffs and alcohol supply increased, the mortality rose substantially (Nemtsov \& Razvodovsky, 2008). Alternatively, the improvement in mortality in late 1995 coincided with a reduction in alcohol sales outlets (Nemtsov, 2002). The shock in the early 1990s was followed by a period of relative improvement and stability in the middle years of the decade. The subsequent rise in stroke deaths and fatal alcohol poisoning rates from 1998 may be associated with the financial crisis which resulted in hyperinflation, increasing poverty, political and economic uncertainty.

Before concluding, several potential limitations of this study must be mentioned. In particular, there was the risk of omitted variable bias in this work. At a population level, high blood pressure and tobacco use are the most important modifiable risk factors for stroke (Feigin et al., 1998). According to the results of blood pressure measurement, the prevalence of hypertension among Russian men and women was $57 \%$ and $55 \%$ respectively (Laaticainen, 2000). However, the findings highlighting that heavy drinking is associated with increased blood pressure support an alcohol-related hypothesis and suggests that rather than playing major causal role, hypertension may represent a confounding factor (Sesso, Cook, Buring, Manson \& Gaziano, 2008). The high prevalence of smoking among Russian men (about 60\%) probably explains a fact of the high stroke mortality rate among yang middle-aged men compared with the female mortality rate (Bobak,, Gilmore, McKee \& Marmot, 2006). However, use of tobacco products was relatively stable during 1970s-1980s and has fallen substantially in Russia over the 1990s, suggesting that stroke mortality crisis is not a result of a long-term response to smoking trends (Ramstedt, 2009).

Some experts have underlined the importance of to the effect of the psychosocial distress of economic and political reforms as the main reason for the cardiovascular mortality crisis in Russia in the early 1990s (Leon \& Shkolnikov, 2005). In this period Russia faced a deep socioeconomic crisis accompanied by unemployment grows, hyperinflation, dramatic decline in the well being of the majority of the population (Stone, 2000). The turmoil associated with socioeconomic and political transition affected Russian peoples and lead to the relatively high prevalence of depression, anxiety and sleeping disorders that were strongly associated with low socioeconomic status, poor nutrition and adverse health behavior such as binge drinking and smoking (Cockerham et al. 2006, Yukkala et al. 2008). It seems plausible that the psychosocial distress was the main cause of increased demand for alcohol at this time. This demand was met by factors that increased supply following the repeal of state alcohol monopoly in 1992.

In conclusion, the results of present study suggest a positive relation between fatal alcohol poisoning and stroke mortality rates at aggregate level and support the hypothesis that alcohol played a crucial role in cerebrovascular mortality fluctuation in Russia over the past decades. This study also indicates that substantial proportions of stroke deaths in Russia are due to acute effect of binge drinking. The findings from the present study have important implications as regards cerebrovascular mortality prevention indicating that a restrictive alcohol policy can be considered as an effective measure of prevention in countries where higher rate of alcohol consumption and binge drinking pattern.

\section{Conflict of Interests}

Nothing to declare

\section{REFERENCES}

[1] Bobak, M., Gilmore, A., Mckee, M., \& Marmot, M. (2006). 
Changes in smoking prevalence in Russia, 1996-2004. Tobacco Control, 15, 131-135.

[2] Box, G. E. P., \& Jenkins, G. M. (1976). Time Series Analysis: forecasting and control. London. Holden-Day Inc.

[3] Cockerham, W. C., Hinote, B. P., \& Abbott, P. (2006). Psychological distress, gender, and health lifestyles in Belarus, Kazakhstan, Russia, and Ukraine. Social Science \& Medicine, 63, 2381-2394.

[4] Djousse, L., Ellison, R.C., Beiser, A., Scaramucci, A., D'Agostino, R.B., \& Wolf P.A. (2002). Alcohol consumption and risk of ischemic stroke. The Framingham study. Stroke, 33, 907-912.

[5] Donna, G.A., Fisher, M., Macleod M., \& Davis S.M. (2007). Stroke. Lancet, 370, 1-12.

[6] Feigin, V.L., Wiebers, D.O., Nikitin, Y.P., O'Fallon, W.M., \& Whisnant J.P. (1998). Risk factors for ischemic stroke in a Russian community. A population-based case-control study. Stroke, 29, 33-39.

[7] Ginter, E. (1995). Cardiovascular risk factors in the former communist countries. Analysis of 40 European MONICA populations. European Journal of Epidemiology, 11, 199-205.

[8] Gorelic P.B. (1987). Alcohol and stroke. Stroke, 18 (1), 268-270.

[9] Hillbom, M., Numminen, H., \& Juvela S. (1999). Recent heavy drinking of alcohol and embolic stroke. Stroke, 30, 2307-2312.

[10] Kesteloot, H., Sans, S., \& Kromhout, D. (2006). Dynamics of cardiovascular and all-cause mortality in Western and Eastern Europe between 1970 and 2000. European Heart Journal, 27, 107-113.

[11] Kim, A.S., \& Johnston S.C. (2011). Global variation in the relative burden of stroke and ischemic heart disese. Circulation, 124, 314-323.

[12] Laaticainen, T. (2000). Cardiovascular risk in the Republic of Karelia, Russia: comparison of major risk factors with North Karelia. Thesis. Helsinki. National Public Health Institute.

[13] Leon, D. A., \& Shkolnikov, V. M. (2005). Social stress and the Russian mortality crisis. Journal of American Medical Association, 279, 790-91.

[14] Leon, D., Saburova, L., Tomkins, S., Andreev E., Kiryanov N., McKee M. \& Shkolnikov V. (2007). Hazardous alcohol drinking and premature mortality in Russia: a population based case-control study. Lancet, 369, 2001-2009.

[15] Mostofsky, E., Burger, M.R., Schlaug, G., Mukamal, K.J., Rosamond, W.D., \& Mittleman M.A. (2010). Alcohol and acute ischemic stroke onset. The stroke onset study. Stroke, $42,1-4$.

[16] Muller-Nordhorn, J., Binting, S., Roll, S., \& Willich, N. (2008). An update on regional variation in cardiovascular mortality within Europe. European Heart Journal, 29(10):1316-1326

[17] Nemtsov, A. V. (2002). Alcohol-related human losses in Russia in the 1980s and 1990s. Addiction, 97, 1413-1425.
[18] Nemtsov, A.V., \& Razvodovsky, Y.E. (2008). Alcohol situation in Russia, 1980-2005. Social and Clinical Psychiatry, 2, 52-60.

[19] Nilssen, O., Averina, M., Brenn, T., Brox, J., Kalinin, A. \& Archipovski, V. (2005). Alcohol consumption and its relation to risk factors for cardiovascular disease in the north-west of Russia: the Archangelsk study. International Journal of Epidemiology, 34, 781-788.

[20] Norstrom, T., \& Skog, O. J. (2001). Alcohol and mortality: methodological and analytical issue in aggregate analysis. Addiction, 96, 5-17.

[21] Pajak, A., \& Kozela, M. (2012). Cardiovascular disease in Central and Eastern Europe. Public Health Review, 33, 416-435.

[22] Patra, J., Taylor, B., Irving, H., Roerecke, M., Baliunas, D., Mohapatra, S., \& Rehm J. (2010). Alcohol consumption and the risk of morbidity and mortality for different stroke types-a systematic review and meta-analysis. BMC Public Health, 10, 258.

[23] Ramstedt, M. (2009). Fluctuations in male ischemic heart disease mortality in Russia 1959-1998: Assessing the importance of alcohol. Drug and Alcohol Review, 28(4), 390-395.

[24] Razvodovsky, Y.E. (2009). Suiside and fatal alcohol poisoning in Russia, 1956-2005. Drugs: education, prevention and policy, 16 (2), 127-139.

[25] Reynolds, K., Lewis, L.B., Nolen, J.D.L., Kinney, G.L., Sathya, B., \& He J. (2003). Alcohol consumption and risk of stroke. JAMA, 289 (5), 579-588.

[26] Rieks, S., Willich, S.N., Mueller-Riemenschneider F. (2012). Trends in age-standardized and age-specific cerebrovascular mortality in Germany between 1980 and 2009. Cerebrovasc Gis, 34, 368-379.

[27] Romelsjo, A., \& Leifman A. (1999). Associated between alcohol consumption and mortality, myocardial infarction, and stroke in 25 year follow up of 49618 young Swedish men. $B M J, 319,821-822$.

[28] Sacco, R., Elkind, M., Boden-Albala, B., Lin, I.F., Kargman, D.E., Hauser, W.A., Shea, S., \& Paik M.C. (1999). The protective effects of moderate alcohol consumption on ischemic stroke. JAMA, 281 (1), 53-60.

[29] Sesso, H.D., Cook, N.R., Buring, J.E., Manson, J.E., \& Gaziano J.M. (2008). Alcohol consumption and the risk of hypertension in women and men. Hypertension, 51, 1080-1087.

[30] Shkolnikov, V. M., McKee, M., Chervyakov, V. V., \& Kyrianov, N. A. (2002). Is the link between alcohol and cardiovascular death among young Russian men attributable to misclassification of acute alcohol intoxication? Evidence from the city of Izhevsk. Journal of Epidemiology \& Community Health, 56, 171-175.

[31] Stickley, A., Leinsalu, M., Andreew, E., Razvodovsky, Y.E., Vagero, D. \& McKee, M. (2007). Alcohol poisoning in Russia and the countries in the European part of the former Soviet Union, 1970-2002. European Journal of Public Health, 17(5), 444-449.

[32] Stone R. Stress: The invisible hand in Eastern Europe's death 
rates. Science, 2000;288:1732-1733.

[33] Sundell, L., Salomaa, V., Vartiainen, E., Poikolainen, K., \&
Laatikainen T. (2008). Increased stroke risk is related to a binge drinking habit. Stroke, 39, 31-79-3184. 